\title{
Florística e Fitossociologia em Áreas de Cerradão e Mata Mesofítica na Estação Ecológica de Pirapitinga, MG
}

\author{
Rômulo Guimarães Giácomo ${ }^{1}$, Marcos Gervasio Pereira ${ }^{2}$, \\ Daniel Costa de Carvalho ${ }^{3}$, Victor Satiro de Medeiros ${ }^{4}$, Tatiana Dias Gaui ${ }^{5}$ \\ ${ }^{1}$ Diretoria de Áreas Verdes, Companhia Municipal de Limpeza Urbana - COMLURB, Rio de Janeiro/RJ, Brasil \\ ${ }^{2}$ Departamento de Solos, Universidade Federal Rural do Rio de Janeiro - UFRRJ, Seropédica/RJ, Brasil \\ ${ }^{3}$ Programa de Pós-Graduação em Ciências Ambientais e Florestais, \\ Universidade Federal Rural do Rio de Janeiro - UFRRJ, Seropédica/RJ, Brasil \\ ${ }^{4}$ Programa de Pós-Graduação em Engenharia Ambiental, Escola Politécnica, \\ Universidade Federal do Rio de Janeiro - UFRJ, Rio de Janeiro/RJ, Brasil \\ ${ }^{5}$ Instituto Nacional de Pesquisa da Amazônia - INPA, Manaus/AM,Brasil
}

\section{RESUMO}

O objetivo deste trabalho foi avaliar a florística e a fitossociologia das espécies presentes em áreas de cerradão e mata mesofítica na Estação Ecológica de Pirapitinga, MG. Para o levantamento da florística e da fitossociologia, foram marcadas 108 parcelas de $100 \mathrm{~m}^{2}$ cada, amostrando-se todos os indivíduos com circunferência à altura do solo $(\mathrm{CAS}) \geq 16 \mathrm{~cm}$. Foram calculadas a frequência, densidade, dominância, os valores de importância e de cobertura, índices de Shannon e Pielou e similaridade florística através do índice de Sorensen. Nas duas áreas de estudo, foi amostrado um total de 1.716 indivíduos distribuídos em 67 espécies, 60 gêneros e 35 famílias. As famílias mais ricas foram Fabaceae e Vochysiaceae. O cerradão foi a formação vegetal com maior riqueza de espécies (67) e maior densidade, enquanto a área de mata mesofítica foi a que apresentou uma vegetação com maior desenvolvimento das espécies.

Palavras-chave: cerrado lato sensu, floresta natural, unidade de conservação.

\section{Floristics and Phytosociology in Areas of Dense Woodland and Mesophytic Forest in the Pirapitinga Ecological Station, Minas Gerais State, Brazil}

\begin{abstract}
The objective of this study was to evaluate the floristic and phytosociological parameters of the species present in areas of mesophytic forest and dense woodland in the Pirapitinga Ecological Station, Minas Gerais state, Brazil. For the survey of floristic and phytosociological parameters, a total of 108 plots of $100 \mathrm{~m}^{2}$ were implanted, where all individuals with circumference at ground height $(\mathrm{CGH}) \geq 16 \mathrm{~cm}$ were sampled in areas of dense woodland and mesophytic forest. The following parameters were calculated: frequency, density, dominance, values of importance and cover, Shannon and evenness indices, and floristic similarity by the Sorensen index. In both study areas, a total of 1,716 individuals of 67 species, 60 genera, and 35 families were sampled. The richest families were Vochysiaceae and Fabaceae. The dense woodland area presented the highest species richness (67) and the highest density, while the mesophytic forest area presented the greatest species development.
\end{abstract}

Keywords: cerrado latu sensu, natural forest, plant conservation. 


\section{INTRODUÇÃO}

O termo cerrado designa uma vegetação de fisionomia e flora próprias, classificada dentro dos padrões de vegetação do mundo como savana (Eiten, 1994). Muito rico floristicamente, é considerado como a flora mais rica entre as savanas mundiais (Klink, 1996). O cerrado destaca-se com relação à biodiversidade devido a sua grande extensão, heterogeneidade vegetal e por conter trechos das bacias hidrográficas dos rios Tocantins, São Francisco e Prata que são as três maiores da América do Sul (Alho \& Martins, 1995; Klink, 1996).

No cerrado ocorrem espontaneamente 12.356 espécies, sendo que deste total 11.627 pertencem à flora vascular nativa deste bioma (Sano et al., 2008). Do ponto de vista fisionômico, os cerrados apresentam dois extremos: o cerradão, fisionomia na qual predomina o componente arbóreo-arbustivo, e o campo limpo, onde há predomínio do componente herbáceo subarbustivo. As demais fisionomias encontradas - campo sujo, campo cerrado, cerrado sensu stricto - podem ser consideradas ecótonas entre o cerradão e o campo limpo (Coutinho, 1978).

O bioma cerrado ocorre na zona tropical da América do Sul, desde os limites inferiores da Floresta Amazônica até os estados de São Paulo e Paraná na região sul-sudeste do Brasil (Silva, 2007). Abrange também pequenas áreas no leste da Bolívia e no nordeste do Paraguai e se estendia originalmente por mais de 2 milhões de $\mathrm{km}^{2}$ (Oliveira \& Ratter, 1995). É considerado uma das 25 áreas do mundo críticas para conservação (hotspots), devido à riqueza biológica $\mathrm{e}$ à alta pressão antrópica a que vem sendo submetido (Brasil, 2002).

Dentre os diversos componentes que formam o ambiente natural, a vegetação pode ser considerada como um bom indicador não só das condições do meio ambiente como também do estado de conservação dos próprios ecossistemas envolvidos (Dias, 2005). Quando uma lista completa das espécies vegetais de uma área é obtida, cada uma pode ser graduada por algum coeficiente quantitativo a fim de indicar a importância de cada uma em relação às demais. Por meio das análises fitossociológicas horizontal e vertical, a estrutura da comunidade vegetal pode ser representada como um todo e comparada com outras comunidades tanto do ponto de vista da composição de espécies como da abundância de suas populações por estratos (Pereira-Silva et al., 2004).A partir do exposto, o objetivo deste trabalho foi identificar a florística e calcular parâmetros fitossociológicos das espécies presentes nas áreas de cerradão e mata mesofítica na Estação Ecológica de Pirapitinga, MG.

\section{MATERIAL E MÉTODOS}

\section{1. Área de estudo}

O estudo foi realizado na Estação Ecológica de Pirapitinga, Ilha das Marias, que possui uma área aproximada de 1.090 ha e perímetro de $20 \mathrm{~km}$, estando localizada no Reservatório da Usina Hidroelétrica de Três Marias, no Estado de Minas Gerais, no município de Morada Nova de Minas. Está compreendida na confluência do córrego Riachão com o rio São Francisco entre as coordenadas $18^{\circ} 20^{\prime} \mathrm{S}-18^{\circ} 23^{\prime} \mathrm{S}$ e $45^{\circ} 17^{\prime} \mathrm{W}-45^{\circ} 20^{\prime} \mathrm{W}$ (Azevedo et al., 1987).

O reservatório de Três Marias foi construído em 1962, sendo a Estação Ecológica criada em 20 de julho de 1987 pelo Decreto n. 94.656. Desde sua criação não há registros de incêndios florestais na Estação Ecológica de Pirapitinga (Azevedo et al., 1987).

O nível da barragem oscila entre a cota máxima de $568 \mathrm{~m}$ até a mínima de $559 \mathrm{~m}$ acima do nível do mar, sendo que em sua cota máxima a estação assume feição de ilha. A estação ecológica está compreendida entre as altitudes 570 e 630 metros acima do nível do mar e o relevo predominantemente é suave ondulado (Azevedo et al., 1987).

A formação geológica da Estação Ecológica se deu a partir de dois grupos de rochas distintas: os siltitos argilosos micáceos e os depósitos colúvio-eluvionares de natureza detrítico-laterítica. Os solos de maior ocorrência na estação são os Latossolos Vermelhos e Cambissolos Háplicos. O tipo climático, segundo Köppen (1948), é Aw (clima tropical úmido, com estação chuvosa no verão e seca no inverno). O período chuvoso se estende de outubro a março; os meses de dezembro, janeiro e fevereiro constituem-se o trimestre mais chuvoso e os meses de junho, julho e agosto, o trimestre mais seco.

A vegetação na estação é constituída por elementos arbóreos com até aproximadamente 25 metros de altura e densidade variável. Apresenta-se sob quatro feições: 
mata mesofítica, cerradão, cerrado sensu stricto e campo sujo (Azevedo et al., 1987). Na mata mesofítica, ou mata seca, são encontrados indivíduos de porte arbóreo, com altura média aproximada de $18 \mathrm{~m}$, que formam um dossel contínuo e emergente, que pode atingir até aproximadamente $25 \mathrm{~m}$ de altura. $\mathrm{O}$ cerradão apresenta porte superior a $9 \mathrm{~m}$, com quatro estratos distintos: o estrato superior com aproximadamente $15 \mathrm{~m}$, um inferior formado por indivíduos de $8 \mathrm{a} 10 \mathrm{~m}$, um estrato arbustivo com altura máxima de $3 \mathrm{~m}$ e um estrato herbáceo constituído por indivíduos jovens. O cerrado sensu stricto apresenta duas variações. Na primeira, o estado arbustivo é denso, o arbóreo esparso, com altura média dos indivíduos em torno de $6 \mathrm{~m}$. O herbáceo apresenta-se contínuo, dominado por gramíneas e com indivíduos jovens das espécies que compõem os demais estratos. Na segunda, não é possível distinguir o estrato arbóreo do arbustivo, uma vez que os indivíduos arbóreos têm de 6 a $10 \mathrm{~m}$ de altura e os elementos arbustivos são de porte desenvolvido (Azevedo et al., 1987). Já o campo sujo é uma fisionomia herbáceo-arbustiva com arbustos e subarbustos espaçados entre si. Estabelece-se sobre solos rasos que podem apresentar pequenos afloramentos rochosos ou sobre solos mais profundos, mas pouco férteis (EMBRAPA, 2015).

\subsection{Florística e fitossociologia}

Nas áreas de campo sujo foram estabelecidas 8 parcelas e nas áreas de cerrado sensu stricto 11 parcelas de $50 \times 20 \mathrm{~m}\left(1.000 \mathrm{~m}^{2}\right)$. Essas parcelas foram subdivididas em 10 subparcelas de $10 \times 10 \mathrm{~m}\left(100 \mathrm{~m}^{2}\right)$. Todas as parcelas foram distribuídas de forma aleatória na sua respectiva formação vegetal e o acesso ocorreu através da utilização de um aparelho de Sistema de Posicionamento Global (GPS) de navegação. No processamento dos dados considerou-se cada subparcela como sendo uma parcela de $10 \times 10 \mathrm{~m}$. Estas totalizaram 80 e 110 parcelas, somando 0,80 e 1,1 ha para as áreas de campo sujo e cerrado sensu stricto, respectivamente. Todas as parcelas amostradas foram marcadas com fitilho e tomadas as coordenadas geográficas com o auxílio de um GPS de navegação. Dentro de cada parcela foram medidos todos os indivíduos com circunferência à altura do solo $(\mathrm{CAS}) \geq 16 \mathrm{~cm}$, com o auxílio de uma fita métrica. Para efeito de cálculos, essas medidas foram transformadas em diâmetro à altura do solo (DAS) com a divisão do respectivo valor por " $\pi$ ". Todas as árvores amostradas foram etiquetadas com plaquetas de couro sintético, numeradas sequencialmente a lápis.

Para cálculos, embora, por definição, a área basal seja calculada a partir da medição do diâmetro do tronco a 1,30 m acima do nível do solo, adotou-se, neste caso, a medição do DAS. Esta condição se faz necessária devido à tortuosidade dos troncos e às abundantes ramificações das árvores do cerrado, que dificultam as medições à altura do peito (Assunção \& Felfili, 2004). Foram coletados materiais botânicos para fins de comparação com a literatura específica, das espécies não identificadas em campo e materiais botânicos férteis e não férteis. Os materiais coletados foram depositados como coleção testemunho no herbário RBR no Instituto de Biologia da Universidade Federal Rural do Rio de Janeiro, conforme as técnicas usuais em botânica de Mori et al. (1985) e Bridson \& Forman (1998). O sistema de classificação botânica adotado foi baseado no Angiosperm Phylogeny Group III (APG, 2009).

No processamento dos dados de fitossociologia, calculou-se a análise de suficiência amostral, para cada tipo de formação vegetal, com intervalo de confiança de 90\% (Phillip, 1994; Nappo et al., 1999; Felfili \& Silva, 2001). Obteve-se a curva de acumulação de espécies de cada tipo de formação vegetal analisada. Foram calculadas a frequência absoluta, frequência relativa, densidade absoluta, densidade relativa, dominância absoluta, dominância relativa segundo Mueller-Dombois \& Ellenberg (1974). Estes resultados geraram os valores de importância. Calcularam-se os índices de diversidade de Shannon (H') e equabilidade de Pielou (J'). A similaridade florística entre as áreas foi estabelecida pelo critério de presença e ausência utilizando-se o índice de Sorensen (Mueller-Dombois \& Ellenberg, 1974).

Para observar o comportamento das espécies, foram construídas curvas de distribuição dos indivíduos vivos por classes diamétricas com intervalos de $5 \mathrm{~cm}$ e por classes de alturas, com intervalos de $2 \mathrm{~m}$.

\section{RESULTADOS E DISCUSSÃO}

Pela análise de suficiência amostral, encontraram-se valores de erro-padrão percentual de 7,06\% e 6,53\% nas áreas de mata mesofítica e cerradão, respectivamente. 
Este resultado indica que houve suficiência amostral para as duas formações vegetais amostradas. Existe suficiência amostral quando o erro-padrão porcentual é de até 10\% (Felfili \& Silva, 2001).

Ao ser amostrada $50 \%$ da área estudada, quantificaram-se entre $68 \%$ e $73 \%$ do total de espécies encontradas nas áreas de mata mesofítica e cerradão, respectivamente. Verificou-se a estabilização da curva espécie/área, o que indica que a amostragem realizada foi suficiente para representar cada formação vegetal estudada.

Amostraram-se um total de 1.716 indivíduos distribuídos em 67 espécies, 60 gêneros e 35 famílias. Do total de espécies, o cerradão foi a formação vegetal com maior riqueza de espécies (67). $\mathrm{Na}$ área de mata mesofítica verificou-se um total de 60 espécies.Verificou-se a presença das espécies Astronium fraxinifolium, Pouteria cf. gardnerii e Protium heptaphyllum na área de mata mesofítica (Tabela 1) e das espécies Astronium fraxinifoliume Protium heptaphyllum na área de cerradão (Tabela 2). Essas espécies podem estar ameaçadas de extinção, pois estão entre aquelas cujas informações são ainda deficientes, não permitindo enquadrá-las com segurança na condição de ameaçadas (Tabelas 1 e 2).

As 67 espécies encontradas na área de cerradão estão subdivididas em 60 gêneros e 35 famílias, sendo Fabaceae (16), Vochysiaceae (4), Malpighiaceae (4) e Myrtaceae (4), Moraceae (3), Bignoniaceae (3) as famílias com maior riqueza, representando $44,77 \%$ do número total de espécies amostradas (Tabela 2).O número de espécies é superior ao verificado por Siqueira et al. (2006), estudando a caracterização florística da Reserva Particular do Patrimônio Natural em Lagumar, MG, onde esses autores identificaram um total de 34 espécies pertencentes a 23 famílias em área de cerradão. Costa \& Araújo (2001) comparando a vegetação arbórea de cerradão na Reserva do Panga verificaram um total de 93 espécies distribuídas em 41 famílias.

O índice de Shannon foi 3,22 e a equabilidade de Pielou 0,78 . Estes valores são muito próximos aos encontrados por Costa \& Araújo (2001), que verificaram índices de 3,54 e 0,78 em área de cerradão, respectivamente. Estudando a distribuição e a abundância de espécies arbóreas em cerradões com diferentes perturbações no Pantanal, Salis et al. (2006) encontraram valores para o índice de Shannon variando de 2,90 a 3,36 e equabilidade de Pielou de 0,80 a 0,91 em diferentes áreas.
Baseado na literatura verifica-se que o índice de Shannon e a equabilidade de Pielou encontram-se dentro da faixa relatada para esse tipo de vegetação. Os valores encontrados para essas áreas sugerem que as áreas de cerradão possuem alta diversidade, com espécies distribuídas homogeneamente.

Verificou-se que a área basal estimada foi de 22,1057 $\mathrm{m}^{2} \mathrm{ha}^{-1} \mathrm{e}$ a densidade absoluta de aproximadamente 1.371 ind $\mathrm{ha}^{-1}$. Em média, esta área basal foi de $0,0161 \mathrm{~m}^{2} \mathrm{ha}^{-1}$, representando um DAS aproximado de $46,89 \mathrm{~m}^{2} \mathrm{~h}^{-1}$ por indivíduo. As espécies com os maiores valores de densidade absoluta foram Xylopia aromatica, Dyospiros sericea, Pterodon pubescens e Vatairea macrocarpa com respectivamente 204,55, 146,97, 103,03 e 93,94 ind ha ${ }^{-1}$ (Tabela 2).

As espécies com os maiores valores de importância (VI) foram Xylopia aromatica, Pterodon pubescens, Dyospiros sericea, Vatairea macrocarpa, Sclerolobium paniculatum var. subvelutinum, Emmotum nitens, Qualea grandiflora, Hymenaea stigonocarpar, Eriotheca pubescens e Cordieria sessilis, representando $60 \%$ do total do valor de importância (Tabela 2). Nesta área observa-se a presença de um número elevado de árvores mortas, ocupando a quarta posição do valor de importância da área de cerradão, representando $8,07 \%$ do total. Um padrão semelhante foi verificado por Andrade et al. (2002) analisando a vegetação da Área de Relevante Interesse Ecológico (ARIE) Cerrado Pé-de-Gigante. Esses autores observaram em áreas de cerrado denso que as árvores mortas em pé representavam a terceira posição do valor de importância da área. Assunção \& Felfili (2004) também verificaram resultados semelhantes. Esses autores encontraram em áreas de cerrado sensu stricto a segunda posição do VI representado por árvores mortas, sugerindo que o elevado percentual de árvores mortas em uma área pode ser indicativo de perturbações.

Estudando áreas de cerradão em uma unidade de conservação no estado de São Paulo, Pereira-Silva et al. (2004) verificaram que as espécies com os maiores valores de importância foram: Ocotea corymbosa, Xylopia aromatica, Siparuna guianensis, Pterodon pubescens, Casearia arborea, Myrcia língua, Miconia albicans, Copaifera langsdorffii. Em estudos comparando a vegetação arbórea e características edáficas de um cerradão e de um cerrado sensu stricto em áreas adjacentes sobre o solo distrófico no 
Tabela 1. Área basal total em 0,42 ha (AB), densidade absoluta (DA), densidade relativa (DR), frequência absoluta (FA), frequência relativa (FR), dominância absoluta (DOA), dominância relativa (DOR), valor de cobertura (VC) e valor de importância (VI). As espécies amostradas em áreas de Mata Mesofítica em ordem decrescente de VI.

Table 1. Total basal area at $0.42 \mathrm{ha}(\mathrm{AB})$, absolute density (DA), relative density $(\mathrm{RD})$, absolute frequency $(\mathrm{AF})$, relative frequency $(\mathrm{FR})$, absolute dominance $(\mathrm{DOA})$, relative dominance $(\mathrm{DOR})$, value of coverage $(\mathrm{VC})$ and importance value (VI). The species in areas of mata mesofítica followed in order of decreasing VI.

\begin{tabular}{|c|c|c|c|c|c|c|c|c|c|c|}
\hline \multirow[b]{2}{*}{ Espécie } & \multirow[b]{2}{*}{$\mathbf{N i}$} & \multirow{2}{*}{$\begin{array}{c}\mathrm{AB} \\
\mathbf{m}^{2} \\
\mathbf{h a}^{-1}\end{array}$} & \multirow{2}{*}{$\begin{array}{l}\text { DA } \\
\text { ind } \\
\text { ha }^{-1}\end{array}$} & \multirow[t]{2}{*}{ DR } & \multirow{2}{*}{$\begin{array}{c}\text { FA } \\
\%\end{array}$} & \multirow[t]{2}{*}{ FR } & \multirow{2}{*}{$\begin{array}{c}\text { DOA } \\
\begin{array}{c}\mathrm{ABm}^{2} \\
\mathrm{ha}^{-1}\end{array}\end{array}$} & \multirow[t]{2}{*}{ DOR } & \multirow{2}{*}{$\begin{array}{c}\text { VC } \\
\%\end{array}$} & \multirow[t]{2}{*}{ VI } \\
\hline & & & & & & & & & & \\
\hline Xylopia aromatica & 121 & 1,8311 & 288,10 & 14,92 & 80,95 & 9,04 & 4,36 & 11,09 & 26,01 & 35,05 \\
\hline Siparuna quianensis & 121 & 0,5205 & 288,10 & 14,92 & 83,33 & 9,31 & 1,24 & 3,15 & 18,07 & 27,38 \\
\hline $\begin{array}{l}\text { Sclerolobium paniculatum } \\
\text { var. subvelutinum }\end{array}$ & 43 & 2,5930 & 102,38 & 5,30 & 47,62 & 5,32 & 6,17 & 15,70 & 21,00 & 26,32 \\
\hline Dyospiros sericea & 83 & 1,4739 & 197,62 & 10,23 & 61,90 & 6,91 & 3,51 & 8,93 & 19,16 & 26,07 \\
\hline Morta & 51 & 0,9459 & 121,43 & 6,29 & 66,67 & 7,45 & 2,25 & 5,73 & 12,02 & 19,46 \\
\hline Virola sebifera & 57 & 0,5957 & 135,71 & 7,03 & 59,52 & 6,65 & 1,42 & 3,61 & 10,64 & 17,28 \\
\hline Copaifera langsdorffii & 14 & 1,3419 & 33,33 & 1,73 & 19,05 & 2,13 & 3,20 & 8,13 & 9,85 & 11,98 \\
\hline Hymenaea stigonocarpar & 9 & 1,3498 & 21,43 & 1,11 & 16,67 & 1,86 & 3,21 & 8,17 & 9,28 & 11,15 \\
\hline Byrsonima sp. & 17 & 0,6628 & 40,48 & 2,10 & 26,19 & 2,93 & 1,58 & 4,01 & 6,11 & 9,04 \\
\hline Cordieria sessilis & 37 & 0,2169 & 88,10 & 4,56 & 23,81 & 2,66 & 0,52 & 1,31 & 5,88 & 8,54 \\
\hline Emmotum nitens & 20 & 0,3008 & 47,62 & 2,47 & 26,19 & 2,93 & 0,72 & 1,82 & 4,29 & 7,21 \\
\hline Ocotea corymbosa & 15 & 0,2527 & 35,71 & 1,85 & 21,43 & 2,39 & 0,60 & 1,53 & 3,38 & 5,77 \\
\hline Eriotheca pubescens & 17 & 0,2420 & 40,48 & 2,10 & 19,05 & 2,13 & 0,58 & 1,47 & 3,56 & 5,69 \\
\hline Tapirira guianensis & 12 & 0,3030 & 28,57 & 1,48 & 14,29 & 1,60 & 0,72 & 1,83 & 3,31 & 4,91 \\
\hline Vatairea macrocarpa & 10 & 0,2944 & 23,81 & 1,23 & 16,67 & 1,86 & 0,70 & 1,78 & 3,02 & 4,88 \\
\hline Schefflera morototoni & 7 & 0,4350 & 16,67 & 0,86 & 11,90 & 1,33 & 1,04 & 2,63 & 3,50 & 4,83 \\
\hline Hirtella glandulosa & 10 & 0,3707 & 23,81 & 1,23 & 11,90 & 1,33 & 0,88 & 2,25 & 3,48 & 4,81 \\
\hline Pterodon pubescens & 11 & 0,0666 & 26,19 & 1,36 & 26,19 & 2,93 & 0,16 & 0,40 & 1,76 & 4,69 \\
\hline Qualea grandiflora & 12 & 0,1411 & 28,57 & 1,48 & 16,67 & 1,86 & 0,34 & 0,85 & 2,33 & 4,20 \\
\hline Protium heptaphyllum & 14 & 0,0058 & 33,33 & 1,73 & 21,43 & 2,39 & 0,01 & 0,04 & 1,76 & 4,16 \\
\hline Myrcia splendens & 13 & 0,0923 & 30,95 & 1,60 & 14,29 & 1,60 & 0,22 & 0,56 & 2,16 & 3,76 \\
\hline Tabebuia impetiginosa & 7 & 0,1023 & 16,67 & 0,86 & 16,67 & 1,86 & 0,24 & 0,62 & 1,48 & 3,34 \\
\hline Luehea divaricata & 7 & 0,1502 & 16,67 & 0,86 & 11,90 & 1,33 & 0,36 & 0,91 & 1,77 & 3,10 \\
\hline Magonia pubescens & 6 & 0,1926 & 14,29 & 0,74 & 9,52 & 1,06 & 0,46 & 1,17 & 1,91 & 2,97 \\
\hline Salvertia convallariaeodora & 2 & 0,3350 & 4,76 & 0,25 & 4,76 & 0,53 & 0,80 & 2,03 & 2,28 & 2,81 \\
\hline Bowdichia vigilioides & 5 & 0,1259 & 11,90 & 0,62 & 11,90 & 1,33 & 0,30 & 0,76 & 1,38 & 2,71 \\
\hline Tabebuia aurea & 7 & 0,0534 & 16,67 & 0,86 & 11,90 & 1,33 & 0,13 & 0,32 & 1,19 & 2,52 \\
\hline Platypodium elegans & 4 & 0,1976 & 9,52 & 0,49 & 7,14 & 0,80 & 0,47 & 1,20 & 1,69 & 2,49 \\
\hline Cecropia pachystachya & 6 & 0,0985 & 14,29 & 0,74 & 9,52 & 1,06 & 0,23 & 0,60 & 1,34 & 2,40 \\
\hline Curatella americana & 4 & 0,1426 & 9,52 & 0,49 & 7,14 & 0,80 & 0,34 & 0,86 & 1,36 & 2,15 \\
\hline Platymenia reticulata & 3 & 0,1533 & 7,14 & 0,37 & 7,14 & 0,80 & 0,36 & 0,93 & 1,30 & 2,10 \\
\hline Cordia sp. & 5 & 0,1064 & 11,90 & 0,62 & 7,14 & 0,80 & 0,25 & 0,64 & 1,26 & 2,06 \\
\hline Astronium fraxinifolium & 4 & 0,1049 & 9,52 & 0,49 & 7,14 & 0,80 & 0,25 & 0,64 & 1,13 & 1,93 \\
\hline Eugenia dysenterica & 5 & 0,0378 & 11,90 & 0,62 & 9,52 & 1,06 & 0,09 & 0,23 & 0,85 & 1,91 \\
\hline Cardioptalum calophyllum & 5 & 0,0206 & 11,90 & 0,62 & 9,52 & 1,06 & 0,05 & 0,12 & 0,74 & 1,80 \\
\hline Callisthene major & 3 & 0,0936 & 7,14 & 0,37 & 4,76 & 0,53 & 0,22 & 0,57 & 0,94 & 1,47 \\
\hline Heteropterys byrsonimifolia & 5 & 0,0430 & 11,90 & 0,62 & 4,76 & 0,53 & 0,10 & 0,26 & 0,88 & 1,41 \\
\hline Guettarda virbunoides & 3 & 0,0371 & 7,14 & 0,37 & 7,14 & 0,80 & 0,09 & 0,22 & 0,59 & 1,39 \\
\hline Brosimum gandichandii & 3 & 0,0101 & 7,14 & 0,37 & 7,14 & 0,80 & 0,02 & 0,06 & 0,43 & 1,23 \\
\hline Plenckia populnea & 2 & 0,0635 & 4,76 & 0,25 & 4,76 & 0,53 & 0,15 & 0,38 & 0,63 & 1,16 \\
\hline Coussarea hydrangeaefolia & 5 & 0,0272 & 11,90 & 0,62 & 2,38 & 0,27 & 0,06 & 0,16 & 0,78 & 1,05 \\
\hline Pseudobombax longifolium & 3 & 0,0030 & 7,14 & 0,37 & 4,76 & 0,53 & 0,01 & 0,02 & 0,39 & 0,92 \\
\hline Lafoensia pacari & 2 & 0,0464 & 4,76 & 0,25 & 2,38 & 0,27 & 0,11 & 0,28 & 0,53 & 0,79 \\
\hline
\end{tabular}


Tabela 1. Continuação...

Table 1. Continued...

\begin{tabular}{|c|c|c|c|c|c|c|c|c|c|c|}
\hline \multirow[b]{2}{*}{ Espécie } & \multirow[b]{2}{*}{$\mathbf{N i}$} & $\mathbf{A B}$ & DA & DR & FA & FR & DOA & DOR & VC & VI \\
\hline & & $\begin{array}{c}\mathrm{m}^{2} \\
\mathrm{ha}^{-1}\end{array}$ & $\begin{array}{l}\text { ind } \\
h \mathrm{a}^{-1}\end{array}$ & & $\%$ & & $\begin{array}{c}\mathrm{ABm}^{2} \\
\mathrm{ha}^{-1}\end{array}$ & \multicolumn{3}{|c|}{$\%$} \\
\hline Agonandra brasiliensis & 1 & 0,0535 & 2,38 & 0,12 & 2,38 & 0,27 & 0,13 & 0,32 & 0,45 & 0,71 \\
\hline Acosmium dasycarpum & 2 & 0,0236 & 4,76 & 0,25 & 2,38 & 0,27 & 0,06 & 0,14 & 0,39 & 0,66 \\
\hline Roupala montana & 2 & 0,0216 & 4,76 & 0,25 & 2,38 & 0,27 & 0,05 & 0,13 & 0,38 & 0,64 \\
\hline Acacia polyphylla & 2 & 0,0179 & 4,76 & 0,25 & 2,38 & 0,27 & 0,04 & 0,11 & 0,35 & 0,62 \\
\hline Aegiphila cf. sellowiana & 1 & 0,0379 & 2,38 & 0,12 & 2,38 & 0,27 & 0,09 & 0,23 & 0,35 & 0,62 \\
\hline Jacaranda brasiliana & 1 & 0,0357 & 2,38 & 0,12 & 2,38 & 0,27 & 0,09 & 0,22 & 0,34 & 0,61 \\
\hline Dimorphandra mollis & 1 & 0,0306 & 2,38 & 0,12 & 2,38 & 0,27 & 0,07 & 0,19 & 0,31 & 0,57 \\
\hline Hyrtella gracilipes & 2 & 0,0093 & 4,76 & 0,25 & 2,38 & 0,27 & 0,02 & 0,06 & 0,30 & 0,57 \\
\hline Pouteria ramiflora & 1 & 0,0245 & 2,38 & 0,12 & 2,38 & 0,27 & 0,06 & 0,15 & 0,27 & 0,54 \\
\hline Pouteria cf. gardnerii & 1 & 0,0228 & 2,38 & 0,12 & 2,38 & 0,27 & 0,05 & 0,14 & 0,26 & 0,53 \\
\hline Casearia cf. commersoniana & 1 & 0,0140 & 2,38 & 0,12 & 2,38 & 0,27 & 0,03 & 0,09 & 0,21 & 0,47 \\
\hline Connarus suberosus & 1 & 0,0092 & 2,38 & 0,12 & 2,38 & 0,27 & 0,02 & 0,06 & 0,18 & 0,44 \\
\hline Ouratea hexasperma & 1 & 0,0092 & 2,38 & 0,12 & 2,38 & 0,27 & 0,02 & 0,06 & 0,18 & 0,44 \\
\hline Qualea parviflora & 1 & 0,0076 & 2,38 & 0,12 & 2,38 & 0,27 & 0,02 & 0,05 & 0,17 & 0,44 \\
\hline Terminalia fagifolia & 1 & 0,0069 & 2,38 & 0,12 & 2,38 & 0,27 & 0,02 & 0,04 & 0,17 & 0,43 \\
\hline Pera glabrata & 1 & 0,0050 & 2,38 & 0,12 & 2,38 & 0,27 & 0,01 & 0,03 & 0,15 & 0,42 \\
\hline Pseudobombax tomentosum & 1 & 0,0032 & 2,38 & 0,12 & 2,38 & 0,27 & 0,01 & 0,02 & 0,14 & 0,41 \\
\hline Total & 811 & 16,5134 & 1930,95 & 100 & 895,24 & 100 & 39,32 & 100 & 200 & 300 \\
\hline
\end{tabular}

Tabela 2. Área basal total em 0,66 ha (AB),densidade absoluta (DA), densidade relativa (DR), frequência absoluta (FA), frequência relativa (FR), dominância absoluta (DOA), dominância relativa (DOR), valor de cobertura (VC) e valor de importância (VI). As espécies amostradas em áreas de Cerradão seguiram a ordem decrescente de VI.

Table 2. Total basal area in 0.66 ha (AB), absolute density (DA), relative density (RD), absolute frequency (AF), relative frequency (FR), absolute dominance (DOA), relative dominance (DOR), value of coverage (VC) and importance value (VI). The species in areas of Cerradão followed the decreasing order of VI.

\begin{tabular}{|c|c|c|c|c|c|c|c|c|c|c|}
\hline & & $\mathbf{A B}$ & DA & DR & FA & FR & DOA & DOR & VC & VI \\
\hline Espécie & $\mathrm{Ni}$ & $\begin{array}{c}\mathrm{m}^{2} \\
\mathbf{h a}^{-1}\end{array}$ & $\begin{array}{l}\text { ind } \\
\text { ha }^{-1}\end{array}$ & & $\%$ & & $\begin{array}{c}\mathrm{ABm}^{2} \\
\mathrm{ha}^{-1}\end{array}$ & & $\%$ & \\
\hline Xylopia aromatica & 135 & 1,4809 & 204,55 & 14,92 & 71,21 & 9,00 & 2,24 & 10,15 & 25,07 & 34,07 \\
\hline Pterodon pubescens & 68 & 2,0974 & 103,03 & 7,51 & 42,42 & 5,36 & 3,18 & 14,38 & 21,89 & 27,25 \\
\hline Dyospiros sericea & 97 & 1,2658 & 146,97 & 10,72 & 48,48 & 6,13 & 1,92 & 8,68 & 19,39 & 25,52 \\
\hline Morta & 74 & 1,1391 & 112,12 & 8,18 & 65,15 & 8,24 & 1,73 & 7,81 & 15,98 & 24,22 \\
\hline Vatairea macrocarpa & 62 & 1,2041 & 93,94 & 6,85 & 48,48 & 6,13 & 1,82 & 8,25 & 15,10 & 21,23 \\
\hline $\begin{array}{l}\text { Sclerolobium paniculatum } \\
\text { var. subvelutinum }\end{array}$ & 25 & 1,0942 & 37,88 & 2,76 & 28,79 & 3,64 & 1,66 & 7,50 & 10,26 & 13,90 \\
\hline Emmotum nitens & 45 & 0,5892 & 68,18 & 4,97 & 37,88 & 4,79 & 0,89 & 4,04 & 9,01 & 13,80 \\
\hline Qualea grandiflora & 32 & 0,5623 & 48,48 & 3,54 & 33,33 & 4,21 & 0,85 & 3,85 & 7,39 & 11,60 \\
\hline Hymenaea stigonocarpar & 26 & 0,3585 & 39,39 & 2,87 & 25,76 & 3,26 & 0,54 & 2,46 & 5,33 & 8,59 \\
\hline Eriotheca pubescens & 19 & 0,4236 & 28,79 & 2,10 & 27,27 & 3,45 & 0,64 & 2,90 & 5,00 & 8,45 \\
\hline Cordieria sessilis & 37 & 0,1484 & 56,06 & 4,09 & 25,76 & 3,26 & 0,22 & 1,02 & 5,11 & 8,36 \\
\hline Siparuna quianensis & 31 & 0,0824 & 46,97 & 3,43 & 25,76 & 3,26 & 0,12 & 0,56 & 3,99 & 7,25 \\
\hline Copaifera langsdorffii & 13 & 0,3524 & 19,70 & 1,44 & 13,64 & 1,72 & 0,53 & 2,42 & 3,85 & 5,58 \\
\hline Magonia pubescens & 17 & 0,2021 & 25,76 & 1,88 & 15,15 & 1,92 & 0,31 & 1,39 & 3,26 & 5,18 \\
\hline Bowdichia vigilioides & 13 & 0,2402 & 19,70 & 1,44 & 15,15 & 1,92 & 0,36 & 1,65 & 3,08 & 5,00 \\
\hline Virola sebifera & 13 & 0,1202 & 19,70 & 1,44 & 13,64 & 1,72 & 0,18 & 0,82 & 2,26 & 3,98 \\
\hline Heteropterys byrsonimifolia & 14 & 0,0673 & 21,21 & 1,55 & 15,15 & 1,92 & 0,10 & 0,46 & 2,01 & 3,92 \\
\hline Caryocar brasiliense & 5 & 0,3494 & 7,58 & 0,55 & 7,58 & 0,96 & 0,53 & 2,39 & 2,95 & 3,90 \\
\hline Platymenia reticulata & 6 & 0,2790 & 9,09 & 0,66 & 9,09 & 1,15 & 0,42 & 1,91 & 2,57 & 3,72 \\
\hline Roupala montana & 12 & 0,0778 & 18,18 & 1,33 & 12,12 & 1,53 & 0,12 & 0,53 & 1,86 & 3,39 \\
\hline
\end{tabular}


Tabela 2. Continuação...

Table 2. Continued...

\begin{tabular}{|c|c|c|c|c|c|c|c|c|c|c|}
\hline \multirow[b]{2}{*}{ Espécie } & \multirow[b]{2}{*}{$\mathbf{N i}$} & $\mathbf{A B}$ & DA & DR & FA & FR & DOA & DOR & VC & VI \\
\hline & & $\begin{array}{c}\mathrm{m}^{2} \\
\mathrm{ha}^{-1}\end{array}$ & $\begin{array}{l}\text { ind } \\
h^{-1}\end{array}$ & \multicolumn{3}{|c|}{$\%$} & $\begin{array}{c}\mathrm{ABm}^{2} \\
\mathrm{ha}^{-1}\end{array}$ & & \multicolumn{2}{|l|}{$\%$} \\
\hline Dipteryx alata & 5 & 0,2581 & 7,58 & 0,55 & 7,58 & 0,96 & 0,39 & 1,77 & 2,32 & 3,28 \\
\hline Ficus gameleira & 1 & 0,4210 & 1,52 & 0,11 & 1,52 & 0,19 & 0,64 & 2,89 & 3,00 & 3,19 \\
\hline $\begin{array}{l}\text { Stryphnodendron adstrin- } \\
\text { gens }\end{array}$ & 7 & 0,1836 & 10,61 & 0,77 & 9,09 & 1,15 & 0,28 & 1,26 & 2,03 & 3,18 \\
\hline Acosmium dasycarpum & 9 & 0,1125 & 13,64 & 0,99 & 10,61 & 1,34 & 0,17 & 0,77 & 1,77 & 3,11 \\
\hline Tapirira guianensis & 10 & 0,0929 & 15,15 & 1,10 & 9,09 & 1,15 & 0,14 & 0,64 & 1,74 & 2,89 \\
\hline Myrcia splendens & 9 & 0,0660 & 13,64 & 0,99 & 10,61 & 1,34 & 0,10 & 0,45 & 1,45 & 2,79 \\
\hline Eugenia dysenterica & 9 & 0,0307 & 13,64 & 0,99 & 12,12 & 1,53 & 0,05 & 0,21 & 1,21 & 2,74 \\
\hline Dalbergia miscolobium & 6 & 0,1127 & 9,09 & 0,66 & 9,09 & 1,15 & 0,17 & 0,77 & 1,44 & 2,58 \\
\hline Byrsonima sp. & 8 & 0,0678 & 12,12 & 0,88 & 9,09 & 1,15 & 0,10 & 0,46 & 1,35 & 2,50 \\
\hline Pouteria ramiflora & 7 & 0,0768 & 10,61 & 0,77 & 9,09 & 1,15 & 0,12 & 0,53 & 1,30 & 2,45 \\
\hline Ficus catappifolia & 5 & 0,1270 & 7,58 & 0,55 & 7,58 & 0,96 & 0,19 & 0,87 & 1,42 & 2,38 \\
\hline Qualea multiflora & 8 & 0,0273 & 12,12 & 0,88 & 9,09 & 1,15 & 0,04 & 0,19 & 1,07 & 2,22 \\
\hline Styrax ferrugineus & 5 & 0,0908 & 7,58 & 0,55 & 7,58 & 0,96 & 0,14 & 0,62 & 1,17 & 2,13 \\
\hline Hirtella glandulosa & 6 & 0,0572 & 9,09 & 0,66 & 7,58 & 0,96 & 0,09 & 0,39 & 1,06 & 2,01 \\
\hline Annona crassiflora & 5 & 0,0683 & 7,58 & 0,55 & 7,58 & 0,96 & 0,10 & 0,47 & 1,02 & 1,98 \\
\hline Qualea parviflora & 5 & 0,0469 & 7,58 & 0,55 & 7,58 & 0,96 & 0,07 & 0,32 & 0,87 & 1,83 \\
\hline Astronium fraxinifolium & 3 & 0,0876 & 4,55 & 0,33 & 4,55 & 0,57 & 0,13 & 0,60 & 0,93 & 1,51 \\
\hline Terminalia fagifolia & 5 & 0,1024 & 7,58 & 0,55 & 1,52 & 0,19 & 0,16 & 0,70 & 1,25 & 1,45 \\
\hline Dimorphandra mollis & 3 & 0,0502 & 4,55 & 0,33 & 4,55 & 0,57 & 0,08 & 0,34 & 0,68 & 1,25 \\
\hline Byrsonima pachyphylla & 4 & 0,0225 & 6,06 & 0,44 & 4,55 & 0,57 & 0,03 & 0,15 & 0,60 & 1,17 \\
\hline Pera glabrata & 3 & 0,0372 & 4,55 & 0,33 & 4,55 & 0,57 & 0,06 & 0,25 & 0,59 & 1,16 \\
\hline Byrsonima sericea & 3 & 0,0139 & 4,55 & 0,33 & 4,55 & 0,57 & 0,02 & 0,10 & 0,43 & 1,00 \\
\hline Brosimum gandichandii & 3 & 0,0110 & 4,55 & 0,33 & 4,55 & 0,57 & 0,02 & 0,08 & 0,41 & 0,98 \\
\hline Salvertia convallariaeodora & 2 & 0,0494 & 3,03 & 0,22 & 3,03 & 0,38 & 0,07 & 0,34 & 0,56 & 0,94 \\
\hline Erythroxylum suberosus & 3 & 0,0175 & 4,55 & 0,33 & 3,03 & 0,38 & 0,03 & 0,12 & 0,45 & 0,83 \\
\hline Aloysia virgata & 2 & 0,0214 & 3,03 & 0,22 & 3,03 & 0,38 & 0,03 & 0,15 & 0,37 & 0,75 \\
\hline Tabebuia aurea & 2 & 0,0179 & 3,03 & 0,22 & 3,03 & 0,38 & 0,03 & 0,12 & 0,34 & 0,73 \\
\hline Casearia cf. commersoniana & 2 & 0,0052 & 3,03 & 0,22 & 3,03 & 0,38 & 0,01 & 0,04 & 0,26 & 0,64 \\
\hline Plenckia populnea & 2 & 0,0143 & 3,03 & 0,22 & 1,52 & 0,19 & 0,02 & 0,10 & 0,32 & 0,51 \\
\hline Curatella americana & 1 & 0,0263 & 1,52 & 0,11 & 1,52 & 0,19 & 0,04 & 0,18 & 0,29 & 0,48 \\
\hline Enterolobium gummiferum & 1 & 0,0259 & 1,52 & 0,11 & 1,52 & 0,19 & 0,04 & 0,18 & 0,29 & 0,48 \\
\hline Machaerium opacum & 1 & 0,0158 & 1,52 & 0,11 & 1,52 & 0,19 & 0,02 & 0,11 & 0,22 & 0,41 \\
\hline Couepia grandiflora & 1 & 0,0144 & 1,52 & 0,11 & 1,52 & 0,19 & 0,02 & 0,10 & 0,21 & 0,40 \\
\hline Protium heptaphyllum & 1 & 0,0137 & 1,52 & 0,11 & 1,52 & 0,19 & 0,02 & 0,09 & 0,20 & 0,40 \\
\hline Guapira noxia & 1 & 0,0106 & 1,52 & 0,11 & 1,52 & 0,19 & 0,02 & 0,07 & 0,18 & 0,37 \\
\hline Kielmeyera rubriflora & 1 & 0,0106 & 1,52 & 0,11 & 1,52 & 0,19 & 0,02 & 0,07 & 0,18 & 0,37 \\
\hline Machaerium acutifolium & 1 & 0,0097 & 1,52 & 0,11 & 1,52 & 0,19 & 0,01 & 0,07 & 0,18 & 0,37 \\
\hline Rourea induta & 1 & 0,0076 & 1,52 & 0,11 & 1,52 & 0,19 & 0,01 & 0,05 & 0,16 & 0,35 \\
\hline Andira paniculata & 1 & 0,0058 & 1,52 & 0,11 & 1,52 & 0,19 & 0,01 & 0,04 & 0,15 & 0,34 \\
\hline Psidium myrsinoides & 1 & 0,0042 & 1,52 & 0,11 & 1,52 & 0,19 & 0,01 & 0,03 & 0,14 & 0,33 \\
\hline Ocotea corymbosa & 1 & 0,0039 & 1,52 & 0,11 & 1,52 & 0,19 & 0,01 & 0,03 & 0,14 & 0,33 \\
\hline Ouratea hexasperma & 1 & 0,0033 & 1,52 & 0,11 & 1,52 & 0,19 & 0,01 & 0,02 & 0,13 & 0,32 \\
\hline Aspidosperma tomentosum & 1 & 0,0032 & 1,52 & 0,11 & 1,52 & 0,19 & 0,00 & 0,02 & 0,13 & 0,32 \\
\hline Tabebuia ochaceae & 1 & 0,0026 & 1,52 & 0,11 & 1,52 & 0,19 & 0,00 & 0,02 & 0,13 & 0,32 \\
\hline Jacaranda brasiliana & 1 & 0,0020 & 1,52 & 0,11 & 1,52 & 0,19 & 0,00 & 0,01 & 0,12 & 0,32 \\
\hline Pseudobombax longifolium & 1 & 0,0020 & 1,52 & 0,11 & 1,52 & 0,19 & 0,00 & 0,01 & 0,12 & 0,32 \\
\hline Psidium pohlianum & 1 & 0,0020 & 1,52 & 0,11 & 1,52 & 0,19 & 0,00 & 0,01 & 0,12 & 0,32 \\
\hline Schefflera macrocarpa & 1 & 0,0019 & 1,52 & 0,11 & 1,52 & 0,19 & 0,00 & 0,01 & 0,12 & 0,32 \\
\hline Total & 905 & 14,5898 & 1371,21 & 100 & 790,91 & 100 & 22,11 & 100 & 200 & 300 \\
\hline
\end{tabular}


leste do Mato Grosso, Marimon \& Haridasan (2005) observaram que as espécies mais importantes em ordem decrescente de VI foram: Hirtella glandulosa, Sclerolobium paniculatum, Xylopia aromatica, Eriotheca gracilipes e Emmotum nitens, as quais representaram $36,5 \%$ do VI total. Analisando a vegetação da Área de Relevante Interesse Ecológico (ARIE) Cerrado Pé-de-Gigante, Batalha (1997) observou-se em áreas de cerradão que as espécies Anadenanthera falcata, Pterodon pubescens, Copaifera langsdorffii, Pouteria ramiflora Xilopia aromatica eram as que possuíam os maiores valores de importância.

A distribuição diamétrica dos indivíduos vivos da área de cerradão apresentou forma de J-invertido
(Figura 1). Nesta área, pode-se observar o predomínio de indivíduos jovens, pois aproximadamente $75 \%$ do total de indivíduos apresentam DAS de até $15 \mathrm{~cm}$. Nesta área observa-se a tendência a maiores diâmetros e maiores alturas. $\mathrm{Na}$ área de cerradão não foram encontrados indivíduos na classe de altura de 0-2 m, estando a maioria dos indivíduos observados nas classes de 4-6 e 6-8 m, com aproximadamente $62 \%$ do total de indivíduos amostrados (Figura 2).

Em áreas de cerradão Andrade et al. (2002) observaram distribuição do diâmetro dos indivíduos vivos e mortos em formato de J-invertido. Verificaram que $90 \%$ dos indivíduos vivos apresentavam diâmetro máximo de $13 \mathrm{~cm}$ e altura de $5 \mathrm{~m}$, respectivamente.

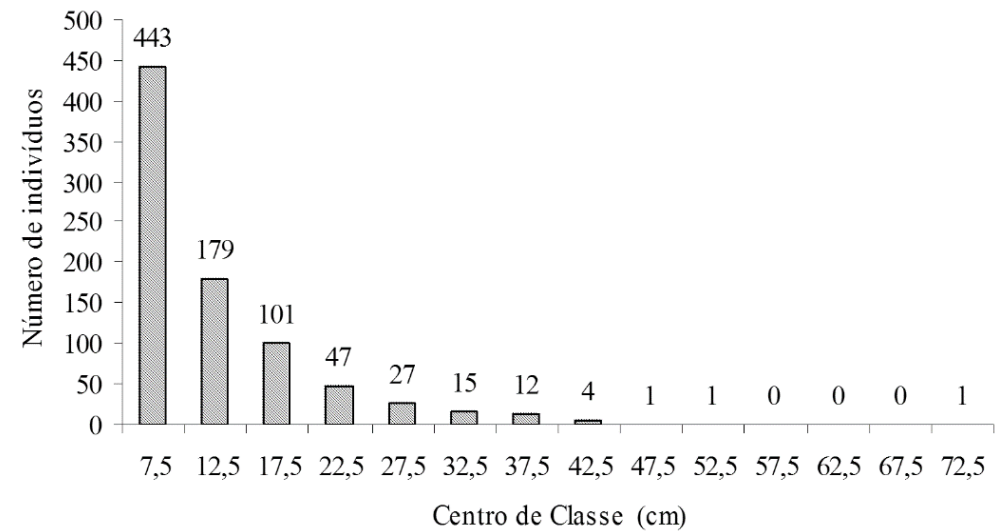

Figura 1. Distribuição dos indivíduos da área de cerradão em classes de diâmetro de $5 \mathrm{~cm}$, na Estação Ecológica de Pirapitinga.

Figure 1. Distribution of individuals in the area of cerradão in diameter classes of $5.0 \mathrm{~cm}$ in diameter, in the Estação Ecológica de Pirapitinga.

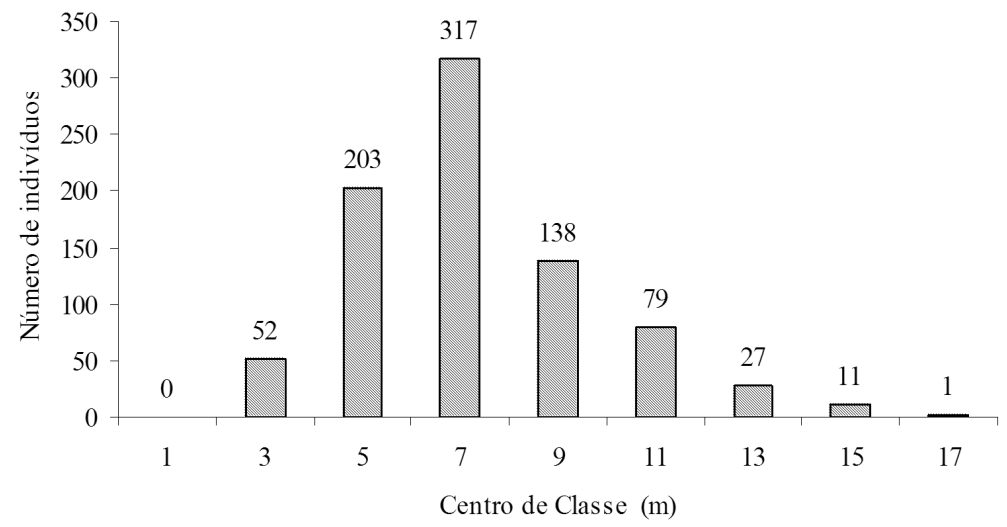

Figura 2. Distribuição dos indivíduos da área de cerradão em classes de altura de $2 \mathrm{~m}$, na Estação Ecológica de Pirapitinga.

Figure 2. Distribution of individuals in the area of cerradão in height classes of $2.0 \mathrm{~m}$, in the Estação Ecológica de Pirapitinga. 
Os autores observaram diâmetro máximo de $44,9 \mathrm{~cm}$ e altura máxima de 9,4 m, concluindo que a área apresentava principalmente indivíduos jovens.

$\mathrm{Na}$ área de mata mesofítica as 60 espécies encontradas estão subdivididas em 55 gêneros e 34 famílias, sendo Fabaceae (11), Vochysiaceae (4), Malvaceae (4), Bignoniaceae (3) e Rubiaceae (3) as famílias mais ricas com $41,66 \%$ do total de espécies amostradas (Tabela 1). Estudando fitossociologia e estrutura diamétrica da mata de galeria do Taquara, na Reserva Ecológica do IBGE em Brasília (Silva, 2004), encontraram-se um total de 110 espécies pertencentes a 91 gêneros e 48 famílias.

O índice de Shannon encontrado foi 3,15 e a equabilidade de Pielou 0,77. Esses valores estão abaixo, mas muito próximos aos verificados na literatura. Guilherme \& Nakajima (2007) estudaram a estrutura da vegetação arbórea de um remanescente ecotonal urbano no Parque do Sabiá, MG. Neste ambiente, esses autores verificaram em floresta estacional valores de 3,70 e 0,83, respectivamente. O valor do índice de Shannon também foi inferior ao observado por Araújo \& Haridasan (1997); Araújo et al. (1997a); Guimarães et al. (2001) em estudos florísticos e fitossociológicos realizados em florestas estacionais da região de Uberlândia, onde a diversidade variou de 3,7 a 4,1. Silva (2004) observou valores de 4,25 e 0,90 para o índice de Shannon e equabilidade de Pielou, respectivamente.

$\mathrm{Na}$ área de mata mesofítica verificou-se área basal estimada de 39,3177 $\mathrm{m}^{2}$ ha $\mathrm{a}^{-1}$ e densidade absoluta de aproximadamente $1.930 \mathrm{ind} \mathrm{ha}^{-1}$. Em média, esta área basal representa em torno de $0,0203 \mathrm{~m}^{2} \mathrm{~h}^{-1}$ por indivíduo, o que corresponde a um DAS de aproximadamente $16,10 \mathrm{~cm}$ por indivíduo. As espécies com as maiores densidades absolutas foram Xylopia aromatica, Siparuna quianensis, Dyospiros sericea, Virola sebifera, Sclerolobium paniculatum var. subvelutinum com 288,10, 288,10, 197,62, $135,71,102,38$ ind $\mathrm{ha}^{-1}$, respectivamente (Tabela 1).

Os valores de área basal e densidade absoluta estão próximos aos verificados por Silva (2004) em áreas de mata de galeria na Reserva Ecológica do IBGE em Brasília.Esses autores quantificaram valores $38,5 \mathrm{~m}^{2} \mathrm{ha}^{-1}$ e 1.573 ind ha ${ }^{-1}$ para área basal e densidade absoluta, respectivamente.

As espécies com os maiores valores de importância (VI) foram Xylopia aromatica, Siparuna quianensis, Sclerolobium paniculatum var. subvelutinum, Dyospiros sericea, Virola sebifera, Copaifera langsdorffi, Hymenaea stigonocarpar, Byrsonima sp, Cordieria sessilis e Emmotum nitens representando $61,94 \%$ do valor de importância total (Tabela 1). Na área de mata mesofítica, também foi verificado um grande número de árvores mortas, representando 6,48\% do VI total (Tabela 1).

A distribuição diamétrica dos indivíduos vivos da área de mata mesofítica também apresentou forma de J-invertido (Figura 3). Na classe de 5-10 cm estão contidas 46,58\% das árvores amostradas na área. Observa-se na área de mata mesofítica que as classes de diâmetro de 10-15 e 15-20 cm são mais significativas que nas demais áreas, representando $26,18 \%$ e $11,84 \%$, respectivamente. Verificou-se que os 30 primeiros

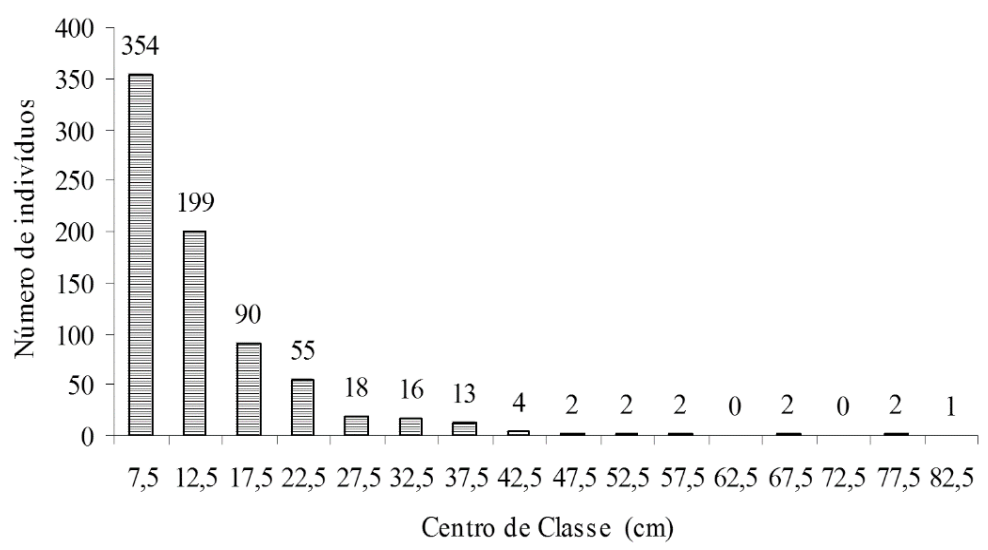

Figura 3. Distribuição dos indivíduos da área de mata mesofítica em classes de diâmetro de $5 \mathrm{~cm}$, na Estação Ecológica de Pirapitinga.

Figure 3. Distribution of individuals in the area of mata mesofítica in classes of $5.0 \mathrm{~cm}$ in diameter, in Estação Ecológica de Pirapitinga. 


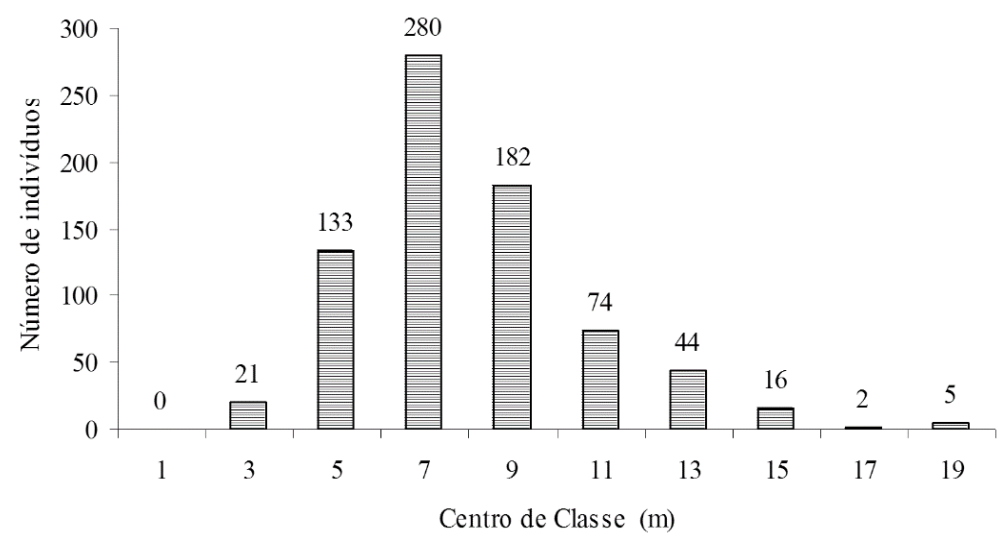

Figura 4. Distribuição dos indivíduos da área de mata mesofítica em classes de altura de 2 m, na Estação Ecológica de Pirapitinga.

Figure 4. Distribution of individuals in the area in height classes of $2.0 \mathrm{~m}$, in Estação Ecológica de Pirapitinga.

centímetros de DAP representam 94,21\% do total de árvores amostradas.

Através da observação da Figura 4, constata-se que a área de mata mesofítica apresenta uma vegetação com maior porte das espécies. Nesta área verifica-se que a maior quantidade de indivíduos está presente nas classes de altura de 6-8 e 8-10 m com aproximadamente 59\% do total das árvores amostradas (Figura 4). Não foram observados indivíduos pertencentes à classe 0-2 m. Estudando mata de galeria, Silva (2004) verificou um padrão similar ao observado neste estudo, onde na classe diamétrica de $5-10 \mathrm{~cm}$ ocorreram $52 \%$ do total de árvores amostradas e 91\% dos indivíduos apresentavam DAP menor que $30 \mathrm{~cm}$. De maneira similar ao verificado neste estudo, esses autores também constataram que a maior árvore ocorria na classe de diâmetro de $85-90 \mathrm{~cm}$.

O valor de similaridade entre as áreas pelo índice de Sorensen foi de 0,63. De acordo com Mueller-Dombois \& Ellenberg (1974), existe similaridade florística quando o valor do índice de Sorensen for superior a 0,25 . De forma geral, os valores de similaridade entre as diferentes formações vegetais podem ser considerados altos. Para Kent \& Coker (1992), valores iguais ou superiores a 0,5 indicam a existência de uma alta similaridade entre as áreas.

Ambas as formações vegetais apresentam em comum as famílias Fabaceae e Vochysiaceae, que são as duas famílias mais ricas em número de espécies. Este padrão também foi observado por Costa \& Araújo (2001). Ao comparar áreas de cerrado sensu stricto com cerradão, esses autores observaram que as famílias Fabaceae, Vochysiaceae e Myrtaceae foram as mais representativas nas duas fitocenoses, concluindo que a presença dessas famílias faz parte da configuração da vegetação dessas comunidades.

Nas duas áreas estudadas há uma densidade considerável de árvores mortas por hectare. Em ambas as áreas a espécie Xilopia aromatica aparece em grande densidade e com elevado valor de importância. Esses resultados podem ser indícios da ocorrência de perturbações a que as áreas foram submetidas no passado. Estudando a estrutura comunitária de vinte áreas de cerrados residuais no município de Urbelândia, MG, Araújo et al. (1997b) observaram que as espécies Matayba guianensis, Miconia albicans, Myrcia rostratae Xylopia aromatica ocorriam com elevada densidade em cerrados alterados.

\section{CONCLUSÕES}

Em ambas as áreas de estudos foi verificado um grande número de árvores mortas, ocupando as primeiras posições. Concluiu-se que esse fato poderia ser indicativo de possíveis perturbações.

A distribuição diamétrica apresentou-se sob forma de J-invertido em ambas as áreas estudadas, indicando predomínio de indivíduos jovens.

Pela análise em classes de altura, constatou-se que a área de mata mesofítica apresenta-se sob a forma de uma vegetação com maior desenvolvimento das espécies. 
O valor de similaridade indicou a existência de alta similaridade entre as áreas estudadas.

\section{STATUS DA SUBMISSÃO}

Recebido: 23 nov., 2013

Aceito: 17 fev., 2015

\section{AUTOR(ES) PARA CORRESPONDÊNCIA}

\section{Marcos Gervasio Pereira}

Departamento de Solos, Universidade Federal

Rural do Rio de Janeiro - UFRRJ,

CEP 23897-900, Seropédica, RJ, Brasil

e-mail: gervasio@ufrrj.br

\section{REFERENNCIAS}

Alho CJR, Martins ES. De grão em grão, o cerrado perde espaço (cerrado, impactos do processo de ocupação). Brasília: WWF; 1995.

Andrade LAZ, Felfili JA, Violatti L. Fitossociologia de uma área de cerrado denso na RECOR-IBGE, Brasília DF. Acta Botanica Brasílica 2002; 16(2): 225-240. http:// dx.doi.org/10.1590/S0102-33062002000200009.

Angiosperm Phylogeny Group - APG. An update of the Angiosperm phylogeny group classification for the orders and families of flowering plants: APG III. Botanical Journal of the Linnean Society 2009; 161(2): 105-121. http://dx.doi. org/10.1111/j.1095-8339.2009.00996.x

Araújo GM, Guimarães AJM, Nakajima JN. Fitossociologia de um remanescente de mata mesófila semidecídua urbana, Bosque John Kennedy, Araguari, MG, Brasil. Revista Brasileira de Botanica 1997a; 20(1): 67-77. http://dx.doi. org/10.1590/S0100-84041997000100007.

Araújo GM, Nunes JJ, Rosa AG, Resente EJ. Estrutura comunitária de vinte áreas de cerrados residuais no município de Urbelândia, MG. Daphne 1997b; 7: 7-14.

Araújo GM, Haridasan M. Estrutura fitossociológica de duas matas mesófilas semidecíduas, em Uberlândia, Triângulo Mineiro. Naturalia 1997; 22: 115-129.

Assunção SL, Felfili JM. Fitossociologia de um fragmento de cerrado sensu stricto na APA do Paranoá, DF, Brasil. Acta Botanica Brasílica 2004; 18(4): 903-909. http://dx.doi. org/10.1590/S0102-33062004000400021.

Azevedo LG, Barbosa AAA, Bedretchuk AC, Oliveira ALC, Gorgonio AS, Siqueira FB et al. Ensaio metodológico de identificação e avaliação de unidades ambientais: a estação ecológica de Pirapitinga, MG. Brasilia: SEMA:EMBRAPACPAC; 1987.58 p.
Batalha MA. Análise da vegetação da ARIE cerrado Pé-deGigante (Santa Rita do Passa Quatro, SP) [dissertação]. São Paulo: Instituto de Biociências, Universidade de São Paulo; 1997.

Brasil. Ministério do Meio Ambiente. Biodiversidade brasileira: avaliação e identificação de áreas prioritárias para conservação, utilização sustentável e repartição de benefícios da biodiversidade brasileira. Brasília: MMA/ SBF; 2002.

Bridson D, Forman L. The herbarium handbook. Kew: Royal Botanical Garden; 1998.

Costa AA, Araújo GM. Comparação da vegetação arbórea de cerradão e de cerrado na reserva do Panga, Urbelândia, Minas Gerais. Acta Botanica Brasílica 2001; 15(1): 63-72. http://dx.doi.org/10.1590/S0102-33062001000100008.

Coutinho LM. O conceito de cerrado. Revista Brasileira de Botanica 1978; 1: 17-23.

Dias AC. Composição florística, fitossociologia, diversidade de espécies arbóreas e comparação de métodos de amostragem na floresta ombrófila densa do Parque Estadual Carlos Botelho / SP - Brasil [tese]. Piracicaba: Escola Superior de Agricultura Luis de Queiroz; 2005.

Eiten G. Vegetação do cerrado. In: Pinto MN, editor. Cerrado: caracterização, ocupação e perspectivas. Brasília: Editora Universidade de Brasília; 1994.

Empresa Brasileira de Pesquisa Agropecuária - EMBRAPA. Tipos de vegetação do bioma cerrado [online]. [citado 2015 jun. 18]. Disponível em: http://www.agencia.cnptia.embrapa. br/Agencia16/AG01/arvore/AG01_23_911200585232.html

Felfili J, Silva MC Jr. Biogeografia do bioma cerrado: estudo fitofisionômico na Chapada do Espigão Mestre do São Francisco. Brasília: Universidade de Brasília; 2001.

Guilherme FAG, Nakajima JN. Estrutura da vegetação arbórea de um remanescente ecotonal urbano florestasavana no Parque do Sabiá, em Urbelândia, MG. Revista Árvore 2007; 31(2): 329-338. http://dx.doi.org/10.1590/ S0100-67622007000200016.

Guimarães AJM, Corrêa GF, Araújo GM. Características da vegetação e do solo em duas comunidades vegetais contíguas no Triângulo Mineiro. Boletim do Herbário Ezechias Paulo Heringer 2001; 7: 113-127.

Kent M, Coker P. Vegetation description analyses. London: Behaven Press; 1992.

Klink CA. Relação entre o desenvolvimento agrícola e a biodiversidade. In: Pereira RC, Nasser LCB, editores. Anais VIII simpósio sobre o cerrado: biodiversidade e produção sustentável de alimentos e fibras nos cerrados; 1996; Brasília. Brasília: Embrapa CPAC; 1996. p. 25-27.

Köppen, W. Climatologia: con un estudio de los climas de la tierra. México: Fondo de Cultura Econômica; 1948. 479 p.

Marimon BH Jr, Haridasan M. Comparação da vegetação arbórea e características edáficas de um cerradão e um 
cerrado sensu stricto em áreas adjacentes sobre solo distrófico no leste de Mato Grosso, Brasil. Acta Botanica Brasílica 2005; 19(4): 913-926. http://dx.doi.org/10.1590/ S0102-33062005000400026.

Mori AS, Mattos-Silva LA, Lisboa G, Coradin L. Manual de manejo do herbário fanerogâmico. 2. ed. Ilhéus: CEPLAC; 1985.

Mueller-Dombois D, Ellenberg H. Aims and methods of vegetation ecology. New York: John Wiley \& Sons; 1974.

Nappo ME, Fontes MAL, Oliveira AT Fo. Suficiência amostral e análise do tamanho de parcelas para o estudo da regeneração natural do sub-bosque de povoamentos homogêneos de Mimosa scabrella Benth., em área minerada, em Poços de Caldas-MG. Revista Árvore 1999; 23: 443-453.

Oliveira AT Fo, Ratter JA. A study of the origin of Central Brazilian forests by the analysis of plant species distribution patterns. Edinburgh Journal of Botany 1995; 52(02): 141194. http://dx.doi.org/10.1017/S0960428600000949.

Pereira-Silva EFL, Santos JE, Kageyama PY, Hardt E. Florística e fitossociologia dos estratos arbustivo e arbóreo de um remanescente de cerradão em uma Unidade de Conservação do Estado de São Paulo. Revista Brasileira de Botanica 2004; 27(3): 533-544. http://dx.doi.org/10.1590/ S0100-84042004000300013.
Phillip MS. Measuring trees and forests. Oxford: CAB International; 1994.

Salis SM, Assis MA, Crispim SMA, Casagrande JC. Distribuição e abundância de espécies arbóreas em cerradões no Pantanal, estado do Mato Grosso do Sul, Brasil. Revista Brasileira de Botanica 2006; 29(3): 339-352. http://dx.doi.org/10.1590/S0100-84042006000300002.

Sano MS, Almeida SP, Ribeiro JF. Cerrado: ecologia e flora. Brasília: Embrapa Informação Tecnológica; 2008. vol. 2.

Silva LCR. Dinâmica de transição e interações entre fitofisionomias florestais e formações vegetacionais abertas do bioma cerrado [dissertação]. Brasília: Departamento de Engenharia Florestal, Faculdade de Tecnologia, Universidade de Brasília; 2007.

Silva MC Jr. Fitossociologia e estrutura diamétrica da mata de galeria do Taquara, na reserva ecológica do IBGE, DF. Revista Árvore 2004; 28(3): 419-428. http://dx.doi. org/10.1590/S0100-67622004000300013.

Siqueira AS, Araújo GM, Schiavini I. Caracterização florística da Reserva Particular do Patrimônio Natural (RPPN) Fazenda Carneiro, Lagamar, MG, Brasil. Biota Neotropica 2006; 6(3): http://dx.doi.org/10.1590/S167606032006000300010 . 